Apidologie, 1973, 4 (1), 25-40.

\title{
ÉTUDE D'UNE POPULATION LOCALE D'ABEILLES DANS LE BASSIN RENNAIS EN 1970-1971
}

\section{Im Jahre 1970-1971 durchgeführte Untersuchungen einer lokalen Bienenpopulation im Raume von Rennes und Umgebung}

\author{
Catherine LEFÈrE-LAFARGUE, J. LOUVEAUX* et J. MESQUIDA \\ Laboratoire de recherches de la chaire de zoologie, I.N.R.A., \\ E.N.S.A., 65, rue de Saint-Brieuc, \\ 35042 Rennes Cedex \\ * Station de recherches sur l'abeille et les insectes sociaux, I.N.R.A., \\ 91440 Bures-sur-Yvette
}

\begin{abstract}
SUMMARY
STUDY OF A LOCAL hoNeybee POPUlation IN RENNES AREA IN 1970-1971

First are described the various methods likely to give an image of the biometrical features of the whole of the honeybee colonies in a definite area. The anthors bring up the results of a survey made in 1970-1971 within a $15 \mathrm{~km}$ cicle centered on the town Rennes (Ille-et-Vilaine). Within this area honeybee colonies were recorted. Samples of honeybees were cut off in 18 apiaries and, on the whole, in 84 colonies, that is $8,75 \%$ of the hives known.

The biometrical investigation of bees relates to the cubital index, the colour, the hairiness and the tongue lengh. It enabled to establish that two thirds of the colonies studied could be considered as belonging to the local black race (Apis mellifica mellifica) and that the last. third was composed of hybrids from old crossings with Apis mellifica ligustica. The hybridization rate is variable. The graphic representation of the results shows a gradual change from the pure mellifica pattern to the ligustica pattern, but this latter was not found in a pure state.

Only such surveys, repeated in different areas, would allow to know the present state of the french live-stock with precision and in which extent it is yet possible to find homogeneous populations represensative of the local ecotypes.
\end{abstract}




\section{RESUME}

Après avoir exposé différentes méthodes susceptibles de donner une image des caractères biométriques de l'ensemble des colonies d'abeilles peuplant un secteur géographique déterminé, les auteurs présentent les résultats d'une enquête effectuée en 1970-1971, en prenant comme base la surface comprise à l'intérieur d'un cercle de $15 \mathrm{~km}$ de rayon et ayant pour centre la ville de Rennes (Ille-et-Vilaine). A l'intérieur de ce cercle, les colonies d'abeilles ont été recensées. Des prélèvements d'abeilles ont été faits dans 18 ruchers et, au total, dans 84 colonies, soit $8,75 \%$ des ruches connues.

L'examen biométrique des abeilles a porté sur l'index cubital, la coloration, la pilosité et la longueur de la langue. Il a permis de constater que les $2 / 3$ des colonies étudiées peuvent être considérées comme appartenant à la race noire locale (Apis mellifica mellifica) et que le $1 / 3$ restant est composé d'hybrides provenant d'anciens croisements avec Apis mellifica ligustica. Le taux d'hybridation est variable. La représentation graphique des résultats montre un glissement progressif du type mellifica pur vers le type ligustica mais ce dernier n'a pas été retrouvé à l'état pur.

Seules des enquêtes de ce type, répétées dans différentes régions, permettraient de connaître avec précision l'état actuel du cheptel français et de savoir dans quelle mesure il est encore possible de trouver des populations homogènes représentatives des écotypes locaux.

\section{INTRODUCTION}

Les conditions traditionnelles de travail des apiculteurs ont subi, au cours des cinquante dernières années, des transformations profondes résultant des modifications apportées au milieu par l'évolution de l'agriculture. D'autre part, les introductions sporadiques de reines appartenant à des races géographiques étrangères ont exercé une influence indiscutable, au moins localement, sur les populations d'abeilles.

Il résulte de cet ensemble de transformations d'ordre écologique une situation confuse dont les apiculteurs ont certainement conscience et qui se traduit par des irrégularités de rendement et par un comportement plus ou moins bien adapté de colonies d'abeilles hétérogènes vis-à-vis d'un milieu en constante évolution. Mal préparés à réaliser eux-mêmes un travail de sélection qui exige des connaissances techniques qu'ils n'ont pas, certains apiculteurs tentent de redresser la situation en introduisant de nouvelles souches dont ils attendent une amélioration de leur production. Ce faisant, ils contribuent par la dissémination de mâles et d'essaims étrangers à la propagation de sujets qui, génétiquement, ne sont pas forcément plus avantageux que les sujets locaux. Quant à l'apiculteur amateur qui ne possède aucun moyen de contrôler son cheptel, il doit subir les hybridations sans d'ailleurs bien comprendre les inconvénients qu'elles peuvent présenter pour son rucher.

Tous ces faits sont connus et ont fait l'objet de nombreuses publications, principalement à l'étranger. Nous pensons superflu d'en faire l'analyse, nous contentant de renvoyer le lecteur au compte-rendu d'une journée d'étude consacrée en 1967 aux problèmes de la sélection des abeilles (LouveAux, 1967). Ce qui, malheureusement, fait encore défaut, c'est une estimation correcte de la situation actuelle du cheptel apicole français. Selon les uns, l'abeille locale 
a totalement disparu, remplacée par une collection d'hybrides variés. Selon d'autres, une telle situation n'est jamais que locale et de nombreux îlots subsistent où il est encore possible de trouver des sujets ne présentant pas de signes d'hybridation. Nous serions tentés de donner raison à ces derniers mais nous pensons que le plus important est encore de vérifier par l'observation dans la nature des hypothèses qui, actuellement, ne reposent que sur des observations fragmentaires et non publiées.

$\mathrm{Si}$, comme nous le pensons, l'absence d'une politique nationale apicole raisonnée ne peut que conduire à une aggravation de l'état actuel, il importe de mettre au point des méthodes permettant de connaître de façon rapide l'état des populations locales d'abeilles. En effet, seule une connaissance précise du degré d'hybridation des colonies d'abeilles dans les différentes régions naturelles permettra d'une part, de sauver les souches locales qui peuvent encore l'être et, d'autre part, de déterminer les zones de production où l'utilisation d'hybrides peut se révéler la seule solution économique valable pour augmenter la production.

Les écologistes disposent pour l'étude des populations de différentes méthodes dont la description figure dans tous les manuels. Notre intention n'est donc pas de les décrire à nouveau mais d'examiner dans quelle mesure elles sont applicables à l'abeille.

Nous connaissons grosso modo la densité des populations en France. Elle est en moyenne de 2 colonies par $\mathrm{km}^{2}$ si bien qu'un recensement précis ne présente pas plus de difficultés, théoriquement, que celui des gros mammifères, par exemple. Ceci dans le cas où l'on se place au niveau de la colonie et non de l'individu. Dans le cas inverse on se trouve en présence de populations environ 20000 à 40000 fois supérieures en nombre. La première question est done de savoir quelle méthode est la meilleure pour avoir une image correcte d'une population d'abeilles, aussi bien du point de vue quantitatif que du point de vue qualitatif.

Nous examinerons tout d'abord les avantages et les inconvénients d'une méthode basée sur la capture d'ouvrières butineuses au niveau des plantes. Une telle méthode a pour avantage théorique de donner une chance égale de capture à toutes les ouvrières, qu'elles appartiennent ou non à ces colonies recensées; par ailleurs, elle peut donner des renseignements d'ordre écologique très variés mais sans doute fort difficiles à interpréter. En fait, sur le terrain, la répartition des abeilles butineuses est très hétérogène. Elle se fait à des niveaux de végétation qui vont des strates les plus basses jusqu'à la cîme des arbres où il est pratiquement impossible de les capturer. D'autre part, les végétaux possèdent une attractivité différentielle pour les abeilles en fonction de leur race; les captures effectuées sur trè fle violet n'auront pas la même valeur indicatrice que celles effectuées sur callune, par exemple. Enfin, nos connais- 
sances sur les communications entre les individus d'une même colonie nous permettent de prévoir l'existence d'une concentration préférentielle des abeilles d'une même ruche en un même lieu.

Pour toutes ces raisons, la méthode des captures sur le terrain au niveau des fleurs ne paraît pas susceptible de fournir facilement une image correcte de la population locale d'abeilles. Elle paraît mieux adaptée à des études écologiques qu'à des études de populations.

Une conclusion analogue serait à tirer de l'utilisation des méthodes de piègeage par attraction sur appâts sucrés.

Le piègeage sexuel des mâles pourrait être utilement envisagé mais il pose encore de nombreux problèmes pratiques. Il n'est pas certain non plus qu'il donne toujours une image correcte de la population locale. Par contre, il a l'avantage de donner une idée très précise de la population sexuellement active. Par ailleurs l'étude biométrique des mâles n'est pas sans avantages du fait de leur haploïdie.

Le recensement des colonies d'abeilles et la prise d'échantillons dans les ruches, qui peut paraître une méthode idéale en raison de la faible densité du cheptel apicole, présente cependant des inconvénients qui proviennent de la nécessité de passer par l'intermédiaire de l'apiculteur, lequel n'est pas toujours connu et, lorsqu'il l'est, peut ne pas être disponible, voire consentant. Ce dernier obstacle peut généralement être vaincu dans la mesure où l'utilité de l'opération peut être démontrée à l'intéressé.

Nous pensons donc finalement que le recensement des colonies suivi du prélèvement d'échantillons d'abeilles représentatifs de la colonie constitue, malgré les aléas qu'il comporte, la meilleure méthode d'étude des populations. Les autres méthodes peuvent constituer un appoint ou apporter des renseignements d'ordre écologique.

L'étude dont il sera question ci-dessous, effectuée à titre expérimental dans le bassin rennais, était davantage destinée à tester la méthode et à rechercher ses imperfections et ses limites qu'à fournir des renseignements exhaustifs. Nous avons cependant jugé intéressant d'en exposer les résultats, tout imparfaits qu'ils soient.

\section{MATÉRIEL ET MÉTHODES}

\section{Choix d'un secteur géographique}

Pour des raisons de commodité, notre choix s'est porté sur la ville de Rennes (Ille-etVilaine) et ses environs immédiats. Prenant la ville pour centre nous avons tracé sur la carte un cercle de $15 \mathrm{~km}$ de rayon et décidé que l'étude porterait uniquement sur la surface ainsi délimitée qui est de $706 \mathrm{~km}^{2}$. 
Recensement des colonies d'abeilles

Pour connaître les propriétaires des ruches situées dans le secteur géographique choisi nous avons fait appel aux déclarations effectuées chaque année à la Direction des Services Vétérinaires du département d'Ille-et-Vilaine. Ces déclarations nous ont permis de connaître, rucher par rucher, le nombre des colonies exploitées.

\section{Choix des ruchers}

Ne pouvant matériellement pas faire des prélèvements d'échantillons d'abeilles dans tous les ruchers recensés nous avons du nous limiter à un certain nombre d'entre eux. Il aurait été souhaitable, pour la rigueur de la méthode, de les tirer au sort. Ce fut malheureusement impossible sur la base des renseignements dont nous disposions. Nous avons donc procédé de façon différente; partant du centre du secteur géographique choisi et selon une spirale plus ou moins régulière nous avons établi une liste des propriétaires de ruches connus. Ceux-ci se sont donc trouvés classés dans un ordre correspondant à l'éloignement de leur domicile du centre du secteur. Comme les ruchers ne sont généralement pas au domicile de l'apiculteur, la répartition géographique des ruchers retenus pour l'enquête s'est trouvée casualisée dans une certaine mesure.

\section{Choix des colonies}

Les 11 premiers propriétaires de ruches figurant sur notre liste furent invités à nous conduire dans leurs ruchers, ce qu'ils firent d'ailleurs avec beaucoup de complaisance. Sur place, le nombre des ruches peuplées fut noté et celles devant faire l'objet d'un prélèvement d'abeilles furent désignées à distance de façon à éviter que l'opérateur ne se laisse in fluencer à l'approche de la ruche par l'aspect des abeilles. Dans chaque rucher la proportion des colonies servant aux prélèvements fut au moins égale à $50 \%$; elle était de $100 \%$ dans les très petits ruchers.

\section{Prélèvement des échantillons d'abeilles}

La ruche, préalablement enfumée par le trou de vol, était ouverte et environ 30 ouvrières étaient prélevées, soit sur le couvre-cadres, soit sur un cadre par brossage dans une boîte grillagée d'une capacité d'environ 1 litre. Cette méthode a déjà été décrite par FrESNAYE (1965).

\section{Examens biométriques}

Au laboratoire les abeilles étaient tuées à l'acétate d'éthyle et examinées aussitôt du point de vue des caractères biométriques suivants : index cubital, pilosité, coloration, longueur

TABL. 1. - Valeurs moyennes et écarts-types retenus comme rêférences pour le classement des colonies (d'après FRESNAYE, 1965 et observations personnelles)

$$
\mathbf{m}=\text { moyenne } \quad \sigma=\text { écart-type }
$$

TAB. 1. - Durchschnittswerte und Streuung als Grundlage für die Klassifizierung der Völker (Nach Fresnaye, 1965 und persönlichen Feststellungen) $\mathrm{m}=$ Durchschnittswerte $\quad \sigma=$ Streuung

\begin{tabular}{|c|c|c|c|c|}
\hline $\begin{array}{c}\text { Caractères } \\
\text { Merkmale }\end{array}$ & $\begin{array}{c}\text { Apis me } \\
\overline{\mathbf{m}}\end{array}$ & $\begin{array}{c}\text { mellifica } \\
\qquad \sigma\end{array}$ & $\begin{array}{c}\text { Apis me } \\
\ldots \mathrm{m}\end{array}$ & $\begin{array}{c}\text { ligustica } \\
\sigma\end{array}$ \\
\hline $\begin{array}{l}\text { Index cubital } \ldots \ldots \ldots \ldots \cdots \cdots \cdots \cdots \\
\text { Cubital Index }\end{array}$ & $1,53-1,99$ & $0,17-0,26$ & $2,17-2,75$ & $0,32-0,50$ \\
\hline $\begin{array}{l}\text { Pilositéf........................ } \\
\text { Überhaar mm }\end{array}$ & $0,41-0,54$ & $0,03-0,09$ & $0,24-0,34$ & $0,02-0,05$ \\
\hline $\begin{array}{l}\text { Coloration } \ldots \ldots \ldots \ldots \ldots \ldots \ldots \ldots \ldots \ldots \\
\text { Farbe } 1-9\end{array}$ & $1,30-2,70$ & $0,32-1,71$ & $6,00-7,73$ & $0,63-2,44$ \\
\hline $\begin{array}{l}\text { Longueur de la langue }(\mathrm{mm}) \ldots \ldots \ldots \ldots \\
\text { Rüssellänge }(\mathrm{mm})\end{array}$ & $6,16-6,56$ & $0,01-0,13$ & $6,31-6,65$ & $0,05-0,20$ \\
\hline
\end{tabular}


de la langue. Les méthodes de mesure étaient celles de FrESNAYE (1965) sauf en ce qui concerne la coloration pour laquelle nous avons adopté l'échelle de notation de 1 à 9 préconisée par GETZE (1964). On a utilisé 30 abeilles pour la mesure de l'index cubital et 10 abeilles prises au hasard pour les autres caractères.

Pour chaque colonie étudiée on a calculé la moyenne et l'écart-type des valeurs obtenues. Les valeurs standard de référence pour les différentes races géographiques ont été empruntées à Fresnaye (1965). Nous avons cependant tenu compte d'observations personnelles effectuées sur des abeilles françaises de race noire et sur des abeilles italiennes de race pure élevées dans un de nos ruchers expérimentaux (Tab. 1).

\section{RÉSULTATS}

\section{Résultats du recensement}

Le nombre des apiculteurs recensés est de 30 . Ils exploitent au total 960 ruches réparties dans 96 ruchers, soit une moyenne de 10 colonies par rucher (fig. 1). La densité moyenne des colonies d'abeilles au $\mathrm{km}^{2}$ est de 1,4 c'est-à-dire qu'elle est inférieure à la moyenne nationale qui est estimée à 2. Ceci n'a rien de surprenant si l'on tient compte de la superficie du secteur purement urbain sans vocation apicole et de celle de la forêt domaniale de Rennes qui n'est pas accessible aux apiculteurs.

\section{Résultats des prélèvements d'abeilles}

Les prélèvements d'échantillons d'abeilles ont été faits dans 18 ruchers. Au total 84, ruches ont fait l'objet d'un prélèvement. Étant donné les résultats du recensement, on constate que $19 \%$ des ruchers connus ont été utilisés pour l'enquête et $8,75 \%$ des colonies (fig. 2). D'une façon générale les ruchers qui ont été utilisés pour l'enquête sont peu importants et très caractéristiques à tous les points de vue d'une apiculture d'amateurs sans grande technicité.

\section{Résultats des examens biométriques}

Les 84, échantillons d'abeilles récoltés ont permis d'examiner 2520 abeilles. Plus de 5000 mesures ont été effectuées. Le tableau 2 résume l'ensemble des résultats obtenus, colonie par colonie et rucher par rucher.

En nous basant sur les valeurs standard moyennes et sur les écarts-types caractéristiques de l'abeille noire (Apis mellifica mellifica) de France et de l'abeille italienne (Apis mellifica ligustica) nous avons procédé au classement des différentes colonies en trois catégories :

$1^{\circ}$ les colonies pour lesquelles tous les caractères mesurés et leur écarttype sont dans les limites des valeurs standard retenues pour l'abeille noire française. 


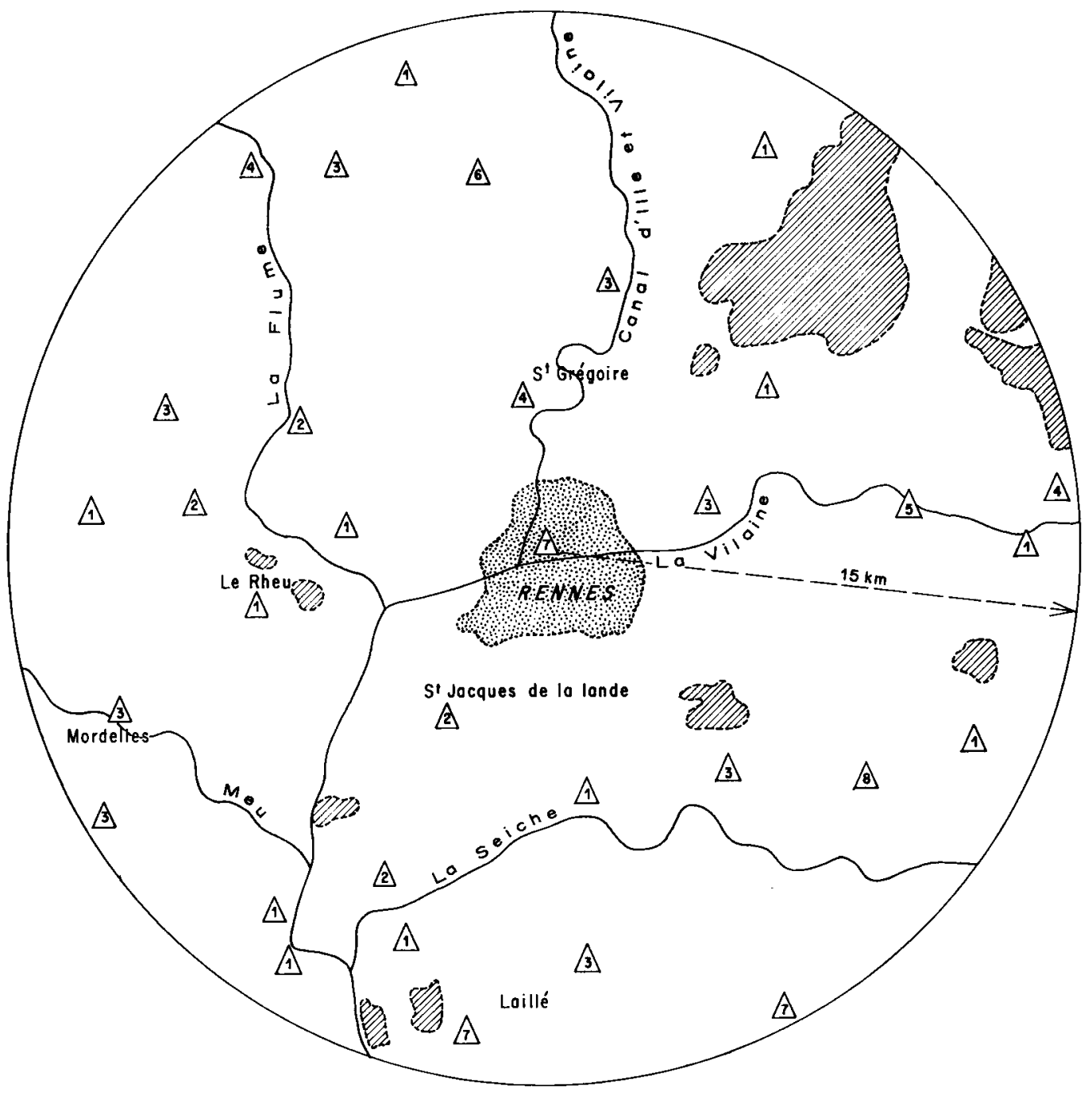

Fic. 1. - Résultats du recensement des ruchers dans le secteur géogaphique choisi.

A l'intérieur de chaque triangle est indiqué le nombre de ruchers exploités sur la commune. Surface pointillée : ville de Rennes.

Surface quadrillé : principaux massifs forestiers.

Aвв. 1. - Ergebnisse der Erfassung der Bienenstände im ausgewählten Landschaftsgebiet.

In jedem Dreieck ist die Zahl der in der Gemeinde festgestellten Bienenstände angegeben.

Die punktierte Fläche : Stadt Rennes.

Schraffierte Fläche : hauptsächlichste Waldbestände. 


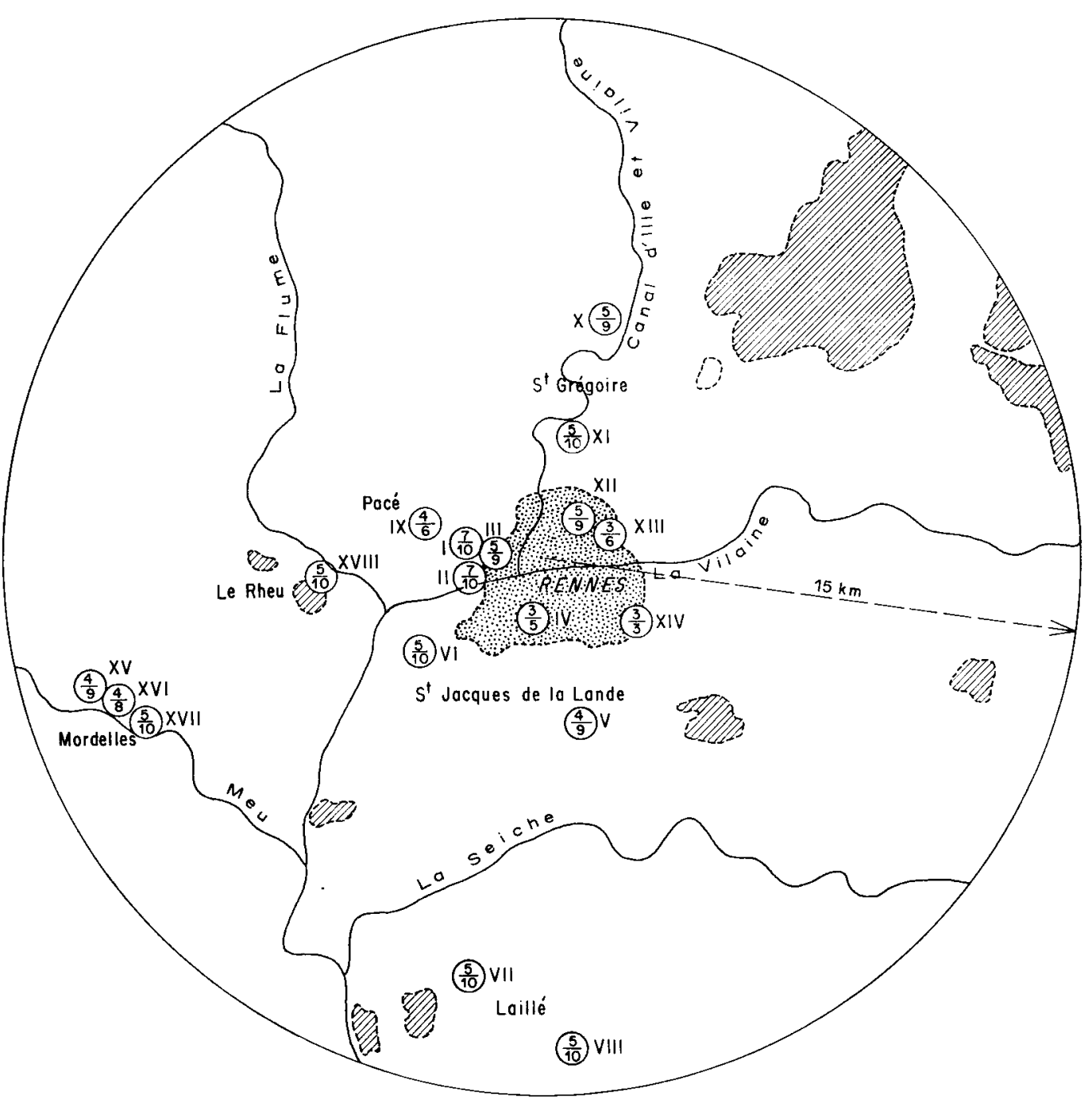

Fig. 2. - Résultats des prélèvements d'abeilles dans le secteur géographique choisi.

A l'intérieur de chaque cercle correspondant à l'un des ruchers étudiés le nombre supérieur est le nombre des colonies dans lesquelles on a fait un prélèvement d'abeilles; le nombre inférieur est le nombre total des colonies du rucher. En chiffres romains la référence du rucher (Voir tableau 2).

Авв. 2. - Ergebnisse der Bienen-Entnahme im Untersuchungsgebiet.

Innerhalb jedes Kreises, der einem der untersuchten Bienenstände entspricht, gibt die obere Ziffer die Zahl der Völker an, von denen Bienen entnommen wurden; die untere Ziffer die Gesamtzahl der Völker des betreffenden Standes. Die römischen Ziffern beziehen sich auf den Bienenstand (s. Tab. 2). 
$2^{\circ}$ les colonies pour lesquelles au moins un caractère ou son écart-type sortent des limites des valeurs standard retenues pour l'abeille noire française et qui, de ce fait, sont à considérer comme présentant des signes d'hybridation.

$3^{\circ}$ les colonies pour lesquelles tous les caractères mesurés et leur écarttype sont dans les limites des valeurs standard retenues pour l'abeille italienne.

Nous n'avons pas mis en évidence de façon suffisamment nette des signes d'hybridation avec des races géographiques autres que ligustica pour qu'il soit utile de faire d'autres catégories.

Les colonies de la première catégorie sont, de loin, les plus nombreuses. On en dénombre 56 contre 28 dans la seconde et 0 dans la $3^{e}$. On peut donc considérer que sur les 84 colonies étudiées $1 / 3$ présentent des signes d'hybridation avec l'abeille italienne; les $2 / 3$ restent dans les limites de l'abeille noire française. Le degré d'hybridation est variable et il faut, pour l'apprécier correctement, examiner successivement les résultats obtenus pour chaque caractère.

\section{a. Index cubital}

Si l'on considère l'ensemble de la population étudiée on constate que son index cubital moyen est de 1,83 avec un écart-type de 0,29 . La distribution autour de la moyenne est assez irrégulière, ce qui indique l'existence de deux groupes qui se chevauchent.

Si l'on sépare la population en deux catégories sur les bases précisées plus haut, on constate que le groupe des colonies ne présentant pas de signes d'hybridation a un index cubital moyen de 1,76 (écart-type 0,23 ), ce qui est en accord complet avec le standard de la race noire. Le groupe des colonies présentant des signes d'hybridation fournit, par contre, un index cubital de 1,96 (écart-type 0,35). L'in fluence de l'abeille italienne est tout-à-fait nette.

\section{b. Pilosité}

Sur l'ensemble de la population étudiée la longueur moyenne de la pilosité est de 0,42 (écart-type 0,06 ). La courbe de distribution tend vers une forme bimodale. En opérant comme pour l'index cubital on trouve une longueur moyenne de 0,44 (écart-type 0,05 ) pour les colonies du $1^{\text {er }}$ groupe et de 0,40 (écart-type 0,19 ) pour les colonies du second groupe. Là encore l'in fluence de l'abeille italienne à pilosité courte est très nette.

\section{c. Coloration}

Sur l'ensemble de la population l'échelle moyenne de la coloration est de 2,89 (écart-type 1,63 ) et la distribution montre un étalement vers les valeurs élevées. Dans le groupe des colonies non hybridées la moyenne est de 2,26 (écart-type 0,88 ). Dans celui des colonies présentant des signes d'hybridation on trouve des valeurs qui vont de 1,37 à 6,62 . 
TABL. 2. - Résultats des examens biométriques des échantillons d'abeilles

$$
\overline{\mathbf{m}}=\text { moyenne } \quad \sigma=\text { écart-type }
$$

Classe 1 : colonies pouvant être considérées comme étant du type mellifica.

Classe 2 : colonies pouvant être considérées comme hybridées.

Les valeurs en caractères gras sont celles qui ont justifié le classement dans la classe 2 .

TAB. 2. - Ergebnisse der biometrischen Untersuchungen der Bienenproben

$$
\overline{\mathbf{m}}=\text { Durchschnittswerte } \quad \sigma=\text { Streuung }
$$

Klasse 1 : Völker, die zum Typus mellifica gerechnet werden können.

Klasse 2 : Völker, die als Hybriden anzusehen sind.

\begin{tabular}{|c|c|c|c|c|c|c|c|c|c|c|}
\hline \multirow{2}{*}{ 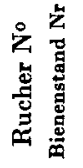 } & \multirow{2}{*}{ 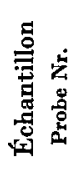 } & \multicolumn{2}{|c|}{$\begin{array}{l}\text { Index cubital } \\
\text { Cubital Index }\end{array}$} & \multicolumn{2}{|c|}{$\begin{array}{l}\text { Pilosité } \\
\text { Utherhaar }\end{array}$} & \multicolumn{2}{|c|}{$\begin{array}{c}\text { Coloration } \\
\text { Farbe }\end{array}$} & \multicolumn{2}{|c|}{$\begin{array}{l}\text { Lg. langue } \\
\text { Rüssellänge }\end{array}$} & \multirow{2}{*}{ 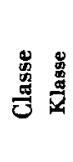 } \\
\hline & & $\overline{\mathbf{m}}$ & $\sigma$ & $\overline{\mathbf{m}}$ & $\sigma$ & $\overline{\mathbf{m}}$ & $\sigma$ & $\overline{\mathbf{m}}$ & $\tau$ & \\
\hline I & $\begin{array}{l}1 \\
2 \\
3 \\
4 \\
5 \\
6 \\
7\end{array}$ & $\begin{array}{l}1,78 \\
1,83 \\
1,71 \\
1,67 \\
1,80 \\
1,99 \\
1,76\end{array}$ & $\begin{array}{l}0,22 \\
0,21 \\
0,23 \\
0,18 \\
0,25 \\
0,24 \\
0,20\end{array}$ & $\begin{array}{l}0,48 \\
0,47 \\
0,51 \\
0,54 \\
0,48 \\
0,45 \\
0,50\end{array}$ & $\begin{array}{l}0,04 \\
0,07 \\
0,04 \\
0,03 \\
0,05 \\
0,03 \\
0,04\end{array}$ & $\begin{array}{l}2,13 \\
1,70 \\
1,50 \\
1,60 \\
2,00 \\
1,70 \\
1,70\end{array}$ & $\begin{array}{l}1,48 \\
0,67 \\
0,53 \\
0,51 \\
0,66 \\
0,48 \\
0,67\end{array}$ & $\begin{array}{l}6,22 \\
6,14 \\
\\
6,14 \\
6,37 \\
6,13 \\
6,22\end{array}$ & $\begin{array}{l}0,50 \\
0,09 \\
\\
0,12 \\
0,23 \\
0,12 \\
0,18\end{array}$ & $\begin{array}{l}1 \\
1 \\
1 \\
1 \\
1 \\
1 \\
1\end{array}$ \\
\hline II & $\begin{array}{r}8 \\
9 \\
10 \\
11 \\
12 \\
13 \\
14\end{array}$ & $\begin{array}{l}1,69 \\
1,71 \\
1,81 \\
1,90 \\
1,66 \\
1,70 \\
1,67\end{array}$ & $\begin{array}{l}0,19 \\
0,26 \\
0,19 \\
0,19 \\
0,17 \\
0,25 \\
0,22\end{array}$ & $\begin{array}{l}0,41 \\
0,43 \\
0,41 \\
0,49 \\
0,49 \\
0,50 \\
0,47\end{array}$ & $\begin{array}{l}0,04 \\
0,04 \\
0,03 \\
0,05 \\
0,04 \\
0,04 \\
0,08\end{array}$ & $\begin{array}{l}1,30 \\
2,50 \\
1,70 \\
2,50 \\
1,90 \\
1,30 \\
2,30\end{array}$ & $\begin{array}{l}0,48 \\
0,85 \\
0,67 \\
1,71 \\
0,57 \\
0,48 \\
0,82\end{array}$ & $\begin{array}{l}6,12 \\
5,98 \\
6,09 \\
6,05 \\
6,05 \\
6,07 \\
6,13\end{array}$ & $\begin{array}{l}0,26 \\
0,21 \\
0,09 \\
0,18 \\
0,11 \\
0,18 \\
0,41\end{array}$ & $\begin{array}{l}1 \\
1 \\
1 \\
1 \\
1 \\
1 \\
1\end{array}$ \\
\hline III & $\begin{array}{l}15 \\
16 \\
17 \\
18 \\
19\end{array}$ & $\begin{array}{l}1,78 \\
1,68 \\
1,94 \\
1,75 \\
1,76\end{array}$ & $\begin{array}{l}0,18 \\
0,21 \\
0,26 \\
0,25 \\
0,19\end{array}$ & $\begin{array}{l}0,44 \\
0,48 \\
0,49 \\
0,47 \\
0,43\end{array}$ & $\begin{array}{l}0,07 \\
0,09 \\
0,03 \\
0,05 \\
0,04\end{array}$ & $\begin{array}{l}2,70 \\
1,60 \\
2,10 \\
1,30 \\
1,89\end{array}$ & $\begin{array}{l}0,48 \\
0,70 \\
1,20 \\
0,67 \\
0,78\end{array}$ & $\begin{array}{l}6,31 \\
6,07 \\
6,03 \\
6,01 \\
5,84\end{array}$ & $\begin{array}{l}0,16 \\
0,18 \\
0,10 \\
0,22 \\
0,20\end{array}$ & $\begin{array}{l}1 \\
1 \\
1 \\
1 \\
1\end{array}$ \\
\hline IV & $\begin{array}{l}20 \\
21 \\
22\end{array}$ & $\begin{array}{l}2,51 \\
1,70 \\
2,08\end{array}$ & $\begin{array}{l}\mathbf{0 , 5 3} \\
0,22 \\
0,30\end{array}$ & $\begin{array}{l}0,39 \\
0,48 \\
0,46\end{array}$ & $\begin{array}{l}0,08 \\
0,03 \\
0,06\end{array}$ & $\begin{array}{l}1,30 \\
1,40 \\
1,90\end{array}$ & $\begin{array}{l}0,48 \\
0,51 \\
1,20\end{array}$ & $\begin{array}{l}6,16 \\
6,13 \\
6,05\end{array}$ & $\begin{array}{l}0,31 \\
0,15 \\
0,17\end{array}$ & $\begin{array}{l}2 \\
1 \\
2\end{array}$ \\
\hline $\mathbf{V}$ & $\begin{array}{l}23 \\
24 \\
25 \\
26\end{array}$ & $\begin{array}{l}1,89 \\
1,88 \\
1,92 \\
1,87\end{array}$ & $\begin{array}{l}\mathbf{0 , 3 3} \\
0,24 \\
0,21 \\
0,22\end{array}$ & $\begin{array}{l}0,46 \\
0,48 \\
0,47 \\
0,46\end{array}$ & $\begin{array}{l}0,05 \\
0,04 \\
0,04 \\
0,04\end{array}$ & $\begin{array}{l}2,75 \\
2,70 \\
3,44 \\
2,70\end{array}$ & $\begin{array}{l}0,90 \\
0,48 \\
1,51 \\
1,77\end{array}$ & $\begin{array}{l}6,16 \\
6,02 \\
6,03 \\
6,00\end{array}$ & $\begin{array}{l}0,11 \\
0,22 \\
0,09 \\
0,14\end{array}$ & $\begin{array}{l}2 \\
1 \\
2 \\
2\end{array}$ \\
\hline VI & $\begin{array}{l}27 \\
28 \\
29 \\
30 \\
31\end{array}$ & $\begin{array}{l}1,94 \\
1,97 \\
1,76 \\
1,77 \\
1,90\end{array}$ & $\begin{array}{l}0,23 \\
0,26 \\
0,22 \\
0,21 \\
0,31\end{array}$ & $\begin{array}{l}0,47 \\
0,44 \\
0,44 \\
0,46 \\
0,45\end{array}$ & $\begin{array}{l}0,04 \\
0,03 \\
0,03 \\
0,05 \\
0,05\end{array}$ & $\begin{array}{l}2,37 \\
3,17 \\
2,77 \\
2,50 \\
3,70\end{array}$ & $\begin{array}{l}0,77 \\
1,15 \\
0,90 \\
0,52 \\
1,53\end{array}$ & $\begin{array}{l}6,13 \\
6,15 \\
6,04 \\
5,93 \\
6,03\end{array}$ & $\begin{array}{l}0,12 \\
0,14 \\
0,16 \\
0,15 \\
0,09\end{array}$ & $\begin{array}{l}1 \\
2 \\
1 \\
1 \\
2\end{array}$ \\
\hline VII & $\begin{array}{l}32 \\
\mathbf{3 3} \\
\mathbf{3 4} \\
\mathbf{3 5} \\
\mathbf{3 6}\end{array}$ & $\begin{array}{l}1,89 \\
1,70 \\
1,85 \\
1,89 \\
1,80\end{array}$ & $\begin{array}{l}0,23 \\
0,20 \\
0,29 \\
0,20 \\
0,23\end{array}$ & $\begin{array}{l}0,47 \\
0,44 \\
0,37 \\
0,45 \\
0,43\end{array}$ & $\begin{array}{l}0,03 \\
0,04 \\
0,06 \\
0,05 \\
0,04\end{array}$ & $\begin{array}{l}2,50 \\
2,20 \\
3,50 \\
2,63 \\
2,60\end{array}$ & $\begin{array}{l}0,53 \\
0,42 \\
1,87 \\
1,27 \\
0,52\end{array}$ & $\begin{array}{l}6,13 \\
5,98 \\
6,21 \\
6,00 \\
6,08\end{array}$ & $\begin{array}{l}0,15 \\
0,16 \\
0,15 \\
0,17 \\
0,13\end{array}$ & $\begin{array}{l}1 \\
1 \\
2 \\
1 \\
\text { I }\end{array}$ \\
\hline
\end{tabular}

Die fett gedruckten Werte sind die, die eine Einstufung in Klasse 2 rechtfertigen. 
Population locale D'ABeilles

\begin{tabular}{|c|c|c|c|c|c|c|c|c|c|c|}
\hline \multirow{2}{*}{ 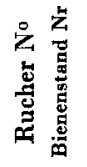 } & \multirow{2}{*}{ 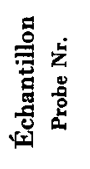 } & \multicolumn{2}{|c|}{$\begin{array}{l}\text { Index cubital } \\
\text { Cubital Index }\end{array}$} & \multicolumn{2}{|c|}{$\begin{array}{l}\text { Pilosité } \\
\text { Überhaar }\end{array}$} & \multicolumn{2}{|c|}{$\begin{array}{c}\text { Coloration } \\
\text { Farbe }\end{array}$} & \multicolumn{2}{|c|}{$\begin{array}{l}\text { Lg. langue } \\
\text { Rüssellange }\end{array}$} & \multirow{2}{*}{ 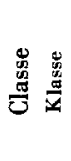 } \\
\hline & & $\overline{\mathbf{m}}$ & 5 & $\overline{\mathbf{m}}$ & $\tau$ & $\overline{\mathbf{m}}$ & $\tau$ & $\overrightarrow{\mathbf{m}}$ & $\tau$ & \\
\hline VIII & $\begin{array}{l}37 \\
38 \\
39 \\
40 \\
41\end{array}$ & $\begin{array}{l}1,69 \\
1,80 \\
1,65 \\
2,00 \\
1,82\end{array}$ & $\begin{array}{l}0,17 \\
0,18 \\
0,22 \\
0,34 \\
0,20\end{array}$ & $\begin{array}{l}0,51 \\
0,43 \\
0,47 \\
0,37 \\
0,44\end{array}$ & $\begin{array}{l}0,02 \\
0,03 \\
0,04 \\
0,03 \\
0,05\end{array}$ & $\begin{array}{l}2,50 \\
2,60 \\
2,90 \\
6,70 \\
2,76\end{array}$ & $\begin{array}{l}0,53 \\
1,43 \\
0,57 \\
1,09 \\
0,79\end{array}$ & $\begin{array}{l}6,15 \\
6,11 \\
6,02 \\
6,37 \\
6,03\end{array}$ & $\begin{array}{l}0,14 \\
0,12 \\
0,21 \\
0,14 \\
0,17\end{array}$ & $\begin{array}{l}1 \\
1 \\
1 \\
2 \\
1\end{array}$ \\
\hline IX & $\begin{array}{l}42 \\
43 \\
44 \\
45\end{array}$ & $\begin{array}{l}1,81 \\
1,78 \\
1,73 \\
1,95\end{array}$ & $\begin{array}{l}0,26 \\
0,23 \\
0,02 \\
0,25\end{array}$ & $\begin{array}{l}0,45 \\
0,40 \\
0,46 \\
0,31\end{array}$ & $\begin{array}{l}0,03 \\
0,03 \\
0,04 \\
0,07\end{array}$ & $\begin{array}{l}2,50 \\
5,40 \\
2,60 \\
5,47\end{array}$ & $\begin{array}{l}0,51 \\
2,17 \\
0,68 \\
2,36\end{array}$ & $\begin{array}{l}6,08 \\
6,24 \\
6,12 \\
6,24\end{array}$ & $\begin{array}{l}0,19 \\
0,12 \\
0,15 \\
0,17\end{array}$ & $\begin{array}{l}1 \\
2 \\
1 \\
2\end{array}$ \\
\hline $\mathbf{X}$ & $\begin{array}{l}46 \\
47 \\
48 \\
49 \\
50\end{array}$ & $\begin{array}{l}1,80 \\
1,72 \\
2,09 \\
1,87 \\
1,82\end{array}$ & $\begin{array}{l}0,22 \\
0,25 \\
0,36 \\
0,26 \\
0,22\end{array}$ & $\begin{array}{l}0,45 \\
0,43 \\
0,40 \\
0,45 \\
0,41\end{array}$ & $\begin{array}{l}0,04 \\
0,03 \\
0,03 \\
0,05 \\
0,02\end{array}$ & $\begin{array}{l}2,53 \\
2,46 \\
3,70 \\
2,10 \\
2,34\end{array}$ & $\begin{array}{l}1,22 \\
1,13 \\
1,60 \\
0,32 \\
0,55\end{array}$ & $\begin{array}{l}6,10 \\
6,10 \\
6,24 \\
6,22 \\
6,22\end{array}$ & $\begin{array}{l}0,11 \\
0,31 \\
0,16 \\
0,09 \\
0,15\end{array}$ & $\begin{array}{l}1 \\
1 \\
2 \\
1 \\
1\end{array}$ \\
\hline XI & $\begin{array}{l}51 \\
52 \\
53 \\
54 \\
55\end{array}$ & $\begin{array}{l}1,96 \\
1,74 \\
2,18 \\
1,96 \\
1,84\end{array}$ & $\begin{array}{l}\mathbf{0 , 3 0} \\
0,20 \\
0,34 \\
0,31 \\
0,20\end{array}$ & $\begin{array}{l}\mathbf{0 , 4 3} \\
\mathbf{0 , 4 3} \\
\mathbf{0 , 3 7} \\
\mathbf{0 , 3 8} \\
\mathbf{0 , 4 4}\end{array}$ & $\begin{array}{l}0,04 \\
0,04 \\
0,06 \\
0,03 \\
0,03\end{array}$ & $\begin{array}{l}4,10 \\
2,26 \\
4,33 \\
3,44 \\
2,22\end{array}$ & $\begin{array}{l}1,84 \\
0,83 \\
1,71 \\
1,66 \\
0,92\end{array}$ & $\begin{array}{l}6,23 \\
6,29 \\
6,27 \\
6,26 \\
6,18\end{array}$ & $\begin{array}{l}0,18 \\
0,05 \\
0,17 \\
0,16 \\
0,11\end{array}$ & $\begin{array}{l}2 \\
1 \\
2 \\
2 \\
1\end{array}$ \\
\hline XII & $\begin{array}{l}56 \\
57 \\
58 \\
59 \\
60\end{array}$ & $\begin{array}{l}1,85 \\
1,72 \\
1,82 \\
1,77 \\
2,02\end{array}$ & $\begin{array}{l}0,17 \\
0,17 \\
0,18 \\
0,17 \\
0,22\end{array}$ & $\begin{array}{l}0,45 \\
0,45 \\
0,45 \\
0,43 \\
0,43\end{array}$ & $\begin{array}{l}0,04 \\
0,03 \\
0,04 \\
0,04 \\
0,06\end{array}$ & $\begin{array}{l}2,07 \\
2,32 \\
2,10 \\
2,10 \\
1,90\end{array}$ & $\begin{array}{l}0,25 \\
0,87 \\
0,32 \\
0,32 \\
0,32\end{array}$ & $\begin{array}{l}6,12 \\
6,04 \\
6,20 \\
6,11 \\
6,13\end{array}$ & $\begin{array}{l}0,10 \\
0,16 \\
0,10 \\
0,07 \\
0,17\end{array}$ & $\begin{array}{l}1 \\
1 \\
1 \\
1 \\
2\end{array}$ \\
\hline XIII & $\begin{array}{l}61 \\
62 \\
63\end{array}$ & $\begin{array}{l}1,71 \\
2,06 \\
1,87\end{array}$ & $\begin{array}{l}0,30 \\
0,24 \\
0,25\end{array}$ & $\begin{array}{l}0,45 \\
0,44 \\
0,41\end{array}$ & $\begin{array}{l}0,05 \\
0,04 \\
0,04\end{array}$ & $\begin{array}{l}2,96 \\
2,10 \\
2,97\end{array}$ & $\begin{array}{l}1,84 \\
0,32 \\
0,89\end{array}$ & $\begin{array}{l}6,21 \\
6,16 \\
6,37\end{array}$ & $\begin{array}{l}0,12 \\
0,08 \\
0,21\end{array}$ & $\begin{array}{l}2 \\
2 \\
2\end{array}$ \\
\hline XIV & $\begin{array}{l}64 \\
65 \\
66\end{array}$ & $\begin{array}{l}1,78 \\
1,77 \\
1,84\end{array}$ & $\begin{array}{l}0,29 \\
0,22 \\
0,26\end{array}$ & $\begin{array}{l}0,44 \\
0,42 \\
0,41\end{array}$ & $\begin{array}{l}0,05 \\
0,04 \\
0,05\end{array}$ & $\begin{array}{l}2,77 \\
3,37 \\
2,50\end{array}$ & $\begin{array}{l}1,23 \\
1,73 \\
0,53\end{array}$ & $\begin{array}{l}6,22 \\
6,29 \\
6,20\end{array}$ & $\begin{array}{l}0,14 \\
0,12 \\
0,10\end{array}$ & $\begin{array}{l}2 \\
2 \\
1\end{array}$ \\
\hline XV & $\begin{array}{l}67 \\
68 \\
69 \\
70\end{array}$ & $\begin{array}{l}1,82 \\
\mathbf{2}, 09 \\
1,75 \\
\mathbf{2 , 4 7}\end{array}$ & $\begin{array}{l}0,17 \\
0,26 \\
\mathbf{0 , 2 7} \\
\mathbf{0 , 3 7}\end{array}$ & $\begin{array}{l}0,41 \\
0,41 \\
0,44 \\
0,37\end{array}$ & $\begin{array}{l}0,03 \\
0,03 \\
0,05 \\
0,07\end{array}$ & $\begin{array}{l}2,24 \\
\mathbf{3 , 3 0} \\
\mathbf{2 , 9 0} \\
\mathbf{5 , 8 0}\end{array}$ & $\begin{array}{l}0,51 \\
1,42 \\
1,67 \\
2,01\end{array}$ & $\begin{array}{l}6,33 \\
6,34 \\
6,29 \\
6,64\end{array}$ & $\begin{array}{l}0,11 \\
0,10 \\
0,12 \\
0,09\end{array}$ & $\begin{array}{l}1 \\
2 \\
2 \\
2\end{array}$ \\
\hline XVI & $\begin{array}{l}71 \\
72 \\
73 \\
74\end{array}$ & $\begin{array}{l}1,56 \\
1,80 \\
1,83 \\
1,99\end{array}$ & $\begin{array}{l}0,25 \\
0,17 \\
0,27 \\
\mathbf{0 , 2 6}\end{array}$ & $\begin{array}{l}0,43 \\
0,46 \\
0,38 \\
0,46\end{array}$ & $\begin{array}{l}0,03 \\
0,03 \\
0,02 \\
0,06\end{array}$ & $\begin{array}{l}2,50 \\
2,10 \\
2,20 \\
2,00\end{array}$ & $\begin{array}{l}0,53 \\
0,32 \\
0,81 \\
0\end{array}$ & $\begin{array}{l}6,26 \\
6,33 \\
6,24 \\
6,25\end{array}$ & $\begin{array}{l}0,13 \\
0,13 \\
0,08 \\
0,11\end{array}$ & $\begin{array}{l}1 \\
1 \\
1 \\
2\end{array}$ \\
\hline XVII & $\begin{array}{l}75 \\
76 \\
77 \\
78 \\
79\end{array}$ & $\begin{array}{l}1,65 \\
1,67 \\
1,80 \\
1,91 \\
1,74\end{array}$ & $\begin{array}{l}0,17 \\
\mathbf{0 , 3 2} \\
0,20 \\
\mathbf{0 , 2 6} \\
0,25\end{array}$ & $\begin{array}{l}0,45 \\
0,42 \\
0,47 \\
0,42 \\
0,35\end{array}$ & $\begin{array}{l}0,04 \\
0,06 \\
0,03 \\
0,04 \\
0,06\end{array}$ & $\begin{array}{l}2,10 \\
2,40 \\
1,80 \\
4,37 \\
2,47\end{array}$ & $\begin{array}{l}0,32 \\
0,52 \\
0,42 \\
2,09 \\
1,41\end{array}$ & $\begin{array}{l}6,15 \\
6,26 \\
6,25 \\
6,25 \\
6,45\end{array}$ & $\begin{array}{l}0,06 \\
\mathbf{0 , 0 9} \\
0,10 \\
0,10 \\
\mathbf{0 , 2 0}\end{array}$ & $\begin{array}{l}1 \\
1 \\
1 \\
2 \\
2\end{array}$ \\
\hline XVIII & $\begin{array}{l}80 \\
81 \mid \\
82 \\
83 \\
84\end{array}$ & $\begin{array}{l}1,83 \\
1,62 \\
1,79 \\
1,98 \\
1,69\end{array}$ & $\begin{array}{l}0,22 \\
0,18 \\
0,21 \\
0,25 \\
0,19\end{array}$ & $\begin{array}{l}0,46 \\
0,46 \\
0,45 \\
0,46 \\
0,47\end{array}$ & $\begin{array}{l}0,04 \\
0,05 \\
0,04 \\
0,05 \\
0,05\end{array}$ & $\begin{array}{l}\mathbf{2 , 1 0} \\
2,30 \\
2,10 \\
\mathbf{3 , 1 6} \\
2,20\end{array}$ & $\begin{array}{l}0,32 \\
0,48 \\
0,32 \\
1,63 \\
0,42\end{array}$ & $\begin{array}{l}6,33 \\
6,10 \\
6,26 \\
6,09 \\
6,16\end{array}$ & $\begin{array}{l}0,16 \\
0,16 \\
0,07 \\
0,20 \\
0,11\end{array}$ & $\begin{array}{l}1 \\
1 \\
1 \\
2 \\
1\end{array}$ \\
\hline
\end{tabular}




\section{d. Longueur de la langue.}

Ce caractère semble, dans notre cas, peu discriminant. Dans aucun cas la longueur de la langue n'a été le caractère permettant le classement d'une colonie dans le groupe des hybrides. La population, considérée dans son ensemble, donne une valeur moyenne de $6,16 \mathrm{~mm}$ (écart-type 0,21 ). Les deux souspopulations sont peu différentes l'une de l'autre : 6,14 (écart-type 0,19 ) pour les colonies noires et 6,23 (écart-type 0,22 ) pour les colonies présentant des signes d'hybridation.

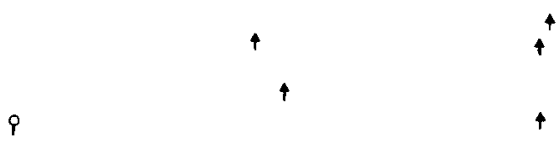

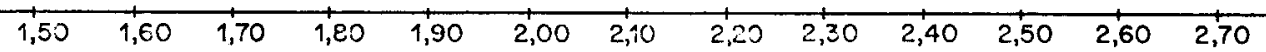

Fig. 3. - Index cubital, coloration et pilosité des 84 colonies étudiées ainsi que de quelques colonies ligustica et mellifica $x$ ligustica prises en dehors du secteur géographique choisi.

En abscisses : index cubital (moyennes).

En ordonnées : coloration dans l'échelle de Gotze (moyennes) :

- - colonies de la classe 1 (Voir tableau 2),

- colonies de la classe 2 (Voir tableau 2),

$\Delta$ - colonies ligustica de race pure prises comme référence,

$\triangle$ - colonies hybrides de première génération mellifica $\mathbf{x}$ ligustica prise comme référence.

La pilosité est représentée par le petit trait ajouté au signe conventionnel ; tourné vers le bas, pilosité courte (moyenne 0,30 ); tourné vers le haut et oblique, pilosité longue (moyenne 0,40 ).

Aвв. 3. - Cubital-Index, Farbe und Überhaar der 84 untersuchten Völker sowie einiger Ligustica und Mellifica x ligustica-Völker, die von ausserhalb des Untersuchungsgebietes stammen.

Abszisse : Cubital-Index (Durchschnitt).

Ordinate : Farbe nach Gotze (Durchschnitt) :

- - Völker der Klasse 1 (s. Tab. 2),

- Völker der Klasse 2 (s. Tab. 2),

$\Delta$ - reinrassige Ligustica-Völker zum Vergleich,

$\triangle$ tu Bastardvölker der 1. Generation mellifica $x$ ligustica zum Vergleich.

Das Utberhaar wird dargestellt durch den kleinen, dem herkömmlichen Zeichen angefügten Strich; nach unten weisend : kurzes Uberhaar $(\varnothing: 0,30)$, schräg nach oben weisend : langes Uberhaar $(\varnothing: 0,40)$. 
Considérant que sur les quatre caractères morphologiques étudiés, trois au moins mettaient en évidence de façon nette l'existence de colonies présentant des signes d'hybridation nous avons recherché une méthode permettant de faire graphiquement la synthèse des résultats. La figure 3 qui intègre l'index cubital, la coloration et la pilosité sur un même graphique fournit une intéressante représentation de l'ensemble de la population étudiée. Elle permet de constater un groupement très compact des colonies que nous avons classéés dans le $1^{\mathrm{er}}$ groupe et un glissement des colonies du second groupe vers les colonies italiennes de race pure qui ont été ajoutées à titre indicatif. On notera le caractère très progressif du glissement et la difficulté de décider du caractère hybride ou non de certaines colonies. On peut en conclure que dans de nombreux cas on se trouve en présence d'hybridations anciennes dont il ne reste que des traces peu importantes.

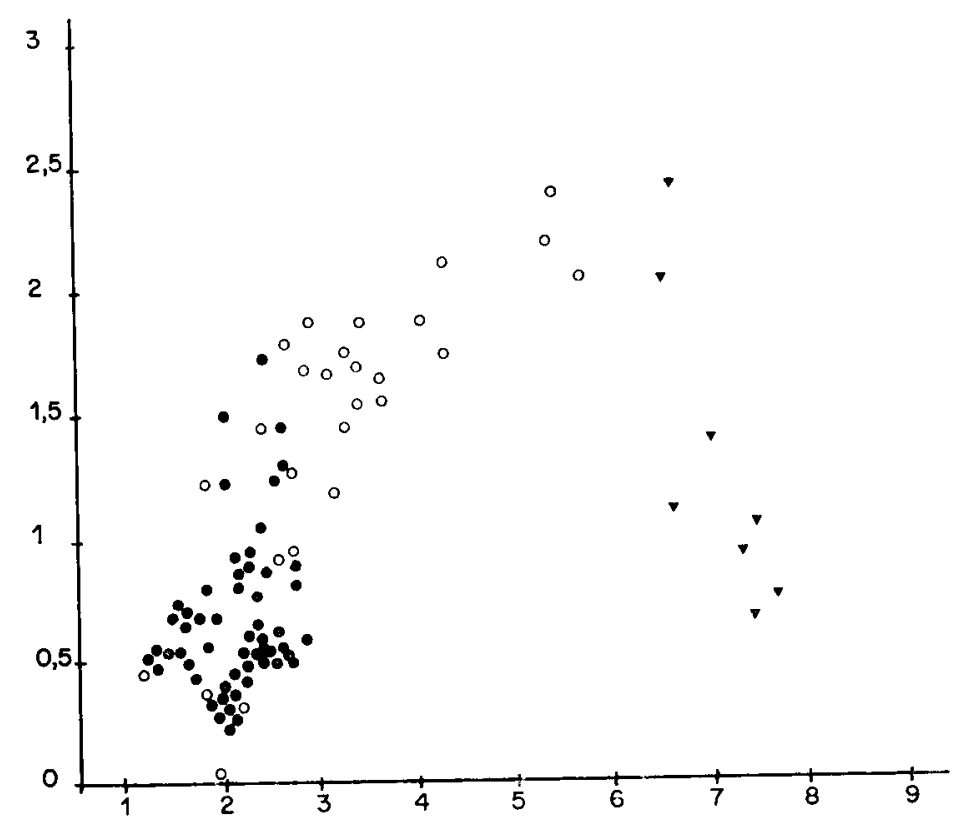

Fig. 4. - Coloration et écart-type de la coloration des 84 colonies étudiées ainsi que de quelques colonies ligustica et mellifica $\mathbf{x}$ ligustica prises en dehors du secteur géographique choisi.

En abscisses : coloration (moyenne) dans l'échelle de Gœtze.

En ordonnées : écarts-types correspondants :

- colonies de la classe 1 (Voir tableau 2),

0 - colonies de la classe 2 (Voir tableau 2),

$\Delta$ - colonies ligustica et mellifica $\mathrm{x}$ ligustica.

Aвв. 4. - Farbe und Farbabweichung der 84, untersuchten Völker sowie einiger von anderer Stelle stammenden Ligustica- und Mellifica $\mathrm{x}$ ligustica Völker.

Abszisse : Farbe nach Gœtze ( $\varnothing$ ).

Ordinate : Abweichtypen entsprechend :

- - Völker der Klasse 1 (s. Tab 2),

- Volker der Klasse 2 (s. Tab. 2),

A - Ligustica und Mellifica $x$ ligustica Völker. 
Il est, par ailleurs, intéressant de représenter graphiquement la variation de l'écart-type d'un caractère déterminé en fonction de la valeur moyenne de ce caractère. C'est ce que nous avons fait pour la coloration (fig. 4). L'augmentation de l'écart-type chez les sujets plus ou moins hybridés est mis nettement en évidence. Chez les abeilles italiennes l'écart-type est d'autant plus faible que la colonie est moins pigmentée. La séparation du groupe des hybrides est d'une grande netteté. Les quelques colonies qui, de toute évidence, ne sont pas à leur place sur le graphique posent un problème mais elles sont trop peu nombreuses pour qu'on puisse l'étudier valablement.

\section{DISCUSSION}

L'étude très limitée que nous avons réalisée dans le bassin rennais nous a permis de mettre en évidence au sein de la population locale d'abeilles, dans des ruchers conduits par des amateurs qui ne sont pas des utilisateurs de reines d'importation, d'un certain taux d'hybridation par l'abeille italienne introduite, très vraisemblablement dans le passé, par des apiculteurs professionnels. L'hybridation avec d'autres races géographiques peut être suspectée mais elle ne semble pas avoir laissé de traces caractéristiques. Seule la ruche $n^{\circ} 20 \mathrm{dont}$ l'index cubital est de 2,5 mais qui est noire et présente une pilosité courte pourrait avoir une ascendance carniolienne.

On trouve dans le même rucher, à la fois des colonies de pure race noire et des hybrides. Le taux d'hybridation apparaît comme étant très difficile à exprimer par un chiffre étant donné le caractère très progressif du passage d'une race à l'autre. Il est cependant permis d'affirmer que dans le secteur géographique étudié les colonies de pure race noire sont les plus nombreuses et que les colonies hybrides ne résultent pas d'un croisement de première génération à de très rares exceptions près.

Il ne semble pas possible de déterminer un centre ou plusieurs centres d'in fluence étrangère. Des ruchers très voisins peuvent présenter des caractères très différents.

Sur le plan pratique, si l'on envisage de multiplier les enquêtes du type de celle que nous avons faite, nous pensons que l'expérience acquise devrait permettre à la fois de simplifier le travail et de le rendre plus significatif.

Au lieu de choisir comme secteur géographique une superficie délimitée par un cercle arbitraire il serait sans doute préférable de considérer une petite région naturelle présentant une certaine unité géographique. Le recensement des ruches devrait se faire de façon aussi complète que possible de telle sorte que le prélèvement des échantillons puisse être parfaitement casualisé. Il conviendrait également que dans chaque rucher la proportion des ruches servant aux prélèvements soit la même. 
Quant aux examens biométriques, ils pourraient être simplifiés compte tenu du fait que certains caractères tels que la longueur de la langue, difficiles à mesurer, sont peu discriminants. En ne conservant que l'index cubital et la coloration il est possible de mettre en évidence une in fluence extérieure du type ligustica. Selon le type d'hybridation recherché on pourra adopter les caractères les plus discriminants et qui peuvent varier d'une région à l'autre.

Ce n'est qu'à ce prix qu'on pourra, en multipliant les enquêtes locales dresser la carte des populations d'abeilles et aborder avec quelque chance de succès le problème de la conservation des écotypes.

Reçu pour publication en novembre 1972.

Eingegangen im November 1972.

\section{REMERCIEMENTS}

Nous adressons nos plus vifs remerciements à M. le Directeur des Services Vétérinaires d'Ille-et-Vilaine ainsi qu'aux apiculteurs qui ont bien voulu collaborer à la réalisation de ce travail.

\section{ZUSAMMENFASSUNG}

Die mehr oder weniger bedeutende Einfuhr von Bienenköniginnen fremder Rassen führt zur Bildung lokaler Populationen, die als Folge ihrer Kreuzung mit den eingeführten Rassen in zunehmendem Masse ihre ursprünglichen Merkmale verlieren. Im allgemeinem ist man nur wenig über die Bedeutung dieses Phänomens unterrichtet. Sehr häufig werden Ansichten verbreitet, die sich nicht auf genaue Beobachtungen gründen. Deshalb haben die Verfasser es sich zur Aufgabe gemacht, einen Weg zu finden, der es erlaubt, die Völker eines bestimmten Raumes zu erfassen und den Grad ihrer Bastardierung durch die eingeführten Rassen zu bestimmen.

Die angewandte Methode besteht darin, eine möglichst vollständige Erfassung aller Bienenvölker in einem begrenzten Landschaftsgebiet durchzuführen und den Bienenvölkern Proben zu entnehmen, die so repräsentativ wie möglich sind. Diese Proben werden biometrisch nach den klassischen Merkmalen : Cubital-Index, Farbe, Überhaar und Rüssellänge untersucht.

In den Jahren 1970 und 1971 wurden in einem Gebiet, das sich im Umkreis von 15 km um die Stadt Rennes (Ille-et-Vilaine) erstreckt, 960 Bienenvölker erfasst, die 30 Imkern gehörten und sich auf 96 Bienenstände verteilten. Die Dichte der Bienenvölker beträgt hier nur 1,4 Völker je km² $^{2}$ gegenüber 2 Völkern je $\mathrm{km}^{2}$ für das gesamte Frankreich. Diese Tatsache erklärt sich aus dem verhältnismässig grossen Anteil des Stadtbezirks von Rennes und des Waldes, der den Imkern nicht zugänglich ist (Abb. 1).

18 Bienenstände (19\% der Gesamtzahl), die praktisch rein zufällig ausgewählt worden waren, wurden besucht und den Völkern 84 Proben von je 30 Bienen entnommen (Abb. 2). Mehr als 5000 biometrische Merkmalsbestimmungen wurden durchgeführt. Sie sind in Tabelle 2 zusammengestellt, die die Durchschnittswerte je Volk und die Streuung aufzeigt.

Grundlage für die Einteilung der Völker in drei Klassen waren die 1965 von FresNaYe für die französische und die Italienerbiene festgelegten Mittelwerte (Tab. 1). 
Die graphische Darstellung der Ergebnisse (Abb. 3 u. 4) zeigt, dass $2 / 3$ der untersuchten Völker biometrische Merkmale aufweisen, die den Normen der schwarzen französischen Biene vollkommen entsprechen. Das restliche Drittel setzt sich aus Hybriden zusammen, die aus früheren Kreuzungen mit Apis mellifica ligustica stammen. Man kann ein fortschreitendes Abgleiten des Typus mellifica zum Typus ligustica feststellen, rein wurde dieser Typus jedoch nicht beobachtet. Diese Erscheinung lässt sich leicht erklären : Keiner der an diesen Untersuchungen beteiligten Imker hat Italiener-Königinnen erworben.

Die Anzeichen einer Bastardierung sind deutlich im Zusammenhang mit den Praktiken von Berufsimkern in der Vergangenheit zu sehen, die bei den Imkern im Untersuchungsgebiet nicht üblich sind.

Die Zufälle bei der Begattung der Königinnen sind die Ursache dafür, dass man in einund demselben Bienenstand gleichzeitig vollkommen reine Mellifica-Völker neben Hybriden findet. In benachbarten Bienenständen kann man sehr unterschiedliche Rassenmerkmale feststellen.

Einzig Untersuchungen dieser Art, in verschiedenen Gegenden in gleicher Weise durchgeführt, würden es ermöglichen, über den derzeitigen Zustand des französischen Bienenbestandes genaue Kenntnis zu erwerben und zu erfahren, wie weit es noch möglich ist, homogene Populationen anzutreffen, die für die lokalen Ökotypen repräsentativ sind.

\section{RÉFÉRENCES BIBLIOGRAPHIQUES}

Fresnaye J., 1965. Étude biométrique de quelques caractères morphologiques de l'abeille noire française (Apis mellifica mellifica). Ann. Abeille 8 (4) : 271-283.

GoEtze G., 1964. Die Honigbiene in natürlicher und künstlicher Zuchtauslese. 212 p., 50 fig., P. Parey. Hambourg.

Louveaux J., 1967. Les problèmes posés par la génétique et la sélection des abeilles. Ann. Abeille 10 (4) : 213-252. 